\title{
The technology of material management and control of the production preparation execution in SMT production line based on digital recognition
}

\author{
Jincheng Cheng ${ }^{1,}$, Aimin Wang ${ }^{1}$ and Yan $\mathrm{Ge}^{1}$ \\ ${ }^{1}$ School of Mechanical Engineering, Beijing Institute of Technology, Beijing 100081, China \\ a493118224@qq.com
}

Keywords: SMT, production preparation, material management, barcode and RFID, experience base

\begin{abstract}
Aimed on the problems of control of the whole SMT production line, the technology of material management and control of the production preparation execution in SMT production line based on digital recognition is proposed. Firstly, the framework of this technology is constructed. Then the three key technologies, which includes the material management technology based on digital recognition, the technology of progressive experience base for material property management, the technology of execution monitoring base on Kanban system etc., are proposed in detail. By means of these technologies above, a more efficient management of the SMT production line by SMT-MES is achieved. Finally, a production management system is designed and verifies the effectiveness of the above technologies successfully.
\end{abstract}

\section{Introduction}

SMT (surface mount technology) currently is one of the most popular technologies in the electronic assembly industry. And in the production of SMT workshop, material management is the most important part of the allocation of resources. With the continuous improvement of the functional requirements of electronic products, the design of the electronic products has gradually become more and more complex corresponding, and the complexity of the structure will make the amount of electronic components in a large number. How to effectively manage these electronic components and to efficiently control and monitor the process of production preparation is always the subject of research.

In the research of the materials management and execution control of production preparation of the SMT production line, there are some inescapable problems: First of all, the materials which need to mount not only have a large number, but also have many kinds, how to avoid material shortage, wrong material, as well as remaining material and so on in material management is an urgent problem to be solved. Secondly, in the process of producing a large number of materials will bring the same amount of a large number of property settings, how to efficiently complete the material properties are the second important issue. Finally, the execution process of production preparation in SMT is quite complex, and the correlation of execution state is very strong, how to monitor and control the process of production efficiently is also an inevitable problem.

Aiming at the problem of material management and control of the production preparation execution in SMT production line, a lot of research has been done at home and abroad. Andy Tseng et al. studied the application of RFID in SMT[1]. Sangchul Kim studied material management system based on daily work [2]. Wang Fan et al. studied the design and implementation of management system in SMT workshop [3]. Zhang Hao et al. do some research on the manufacturing execution system of SMT workshop based on Intelligent Object [4].

Although there are several researches on material management and control of the production preparation execution in SMT production line both at home and abroad, these studies are not perfect. The collection of material turnover information didn't combine with the control of the production preparation execution of the whole order effectively. Because of the quality requirement of electronic products is very high, an effective management and control to the material and 
production process can significantly improve production efficiency and enterprise benefit. The development trend of the future must be the information flow, material flow and business flow of the SMT production process highly unified in the MES (Manufacturing Execution System), which will realize the transparent management of SMT production for the whole process of state, and improve the benefit of the SMT production line efficiency of enterprises.

With the combination of the SMT-MES system designation and the problems discussed above, this paper suggest the technology of material management and control of the production preparation execution in SMT production line based on digital recognition, which is aiming at developing the SMT production line.

\section{The framework of the technology}

The technology of material management and control of the production preparation execution in SMT production line based on digital recognition is mainly to study how to collect and control the order material information, and to monitor the process of production and execution of SMT. This paper will explain the framework of technology with the Fig.1 below.

This paper will combine the two aspects of the business process and key technologies from 1 aspects of the structural framework of this technology.

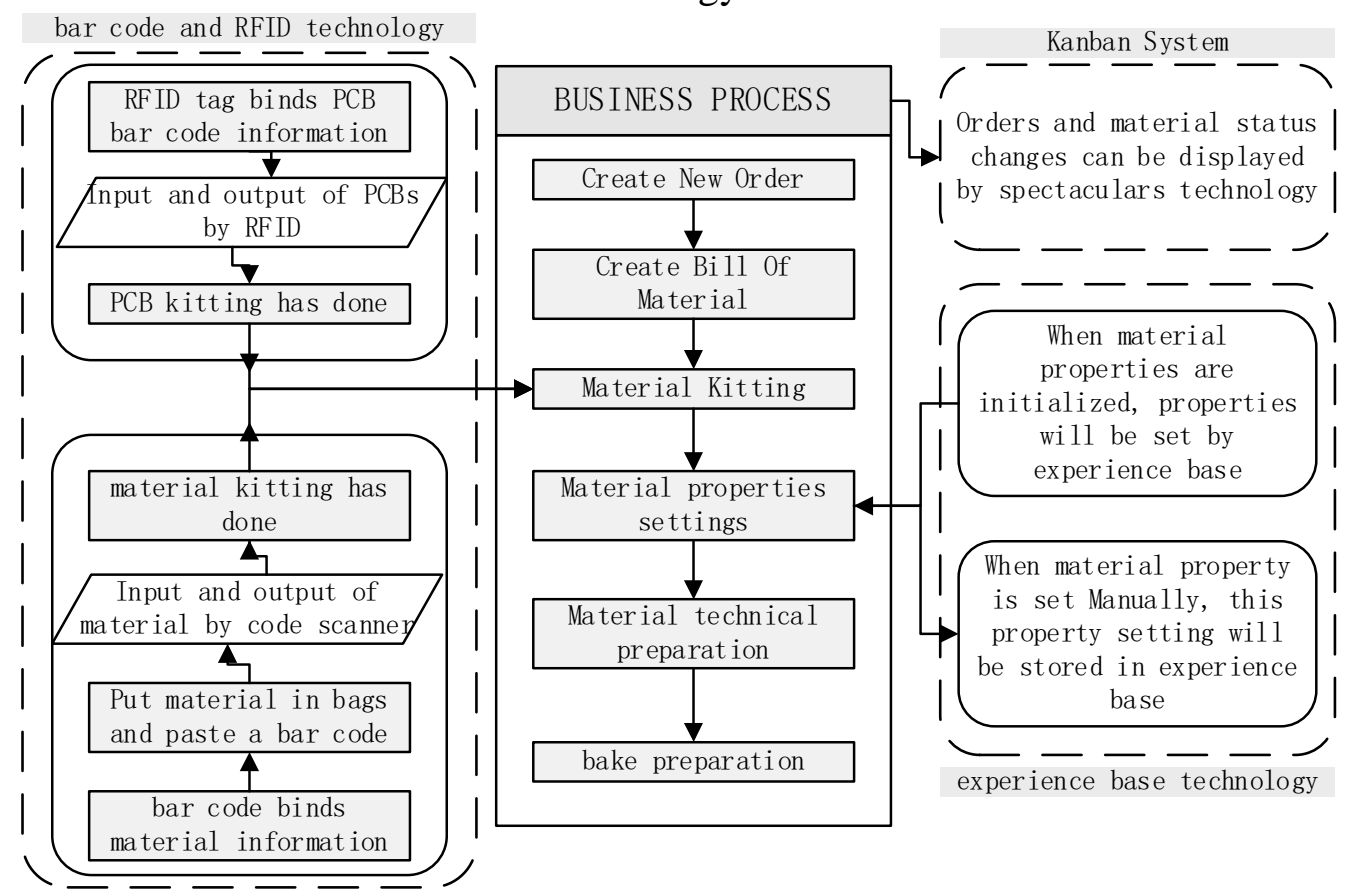

\subsection{The business process of the production preparation in SMT}

Fig.1 The framework of the technology

When a new order begins to be put into operation, the production preparation begins to execute immediately. First of all, the material requirements of each new order should be clear, and manufacturing BOM (Bill of Material) should be created, and the barcode of each material should be created and printed and pasted. Secondly, the input and output of material should be put into operation to meet the order requirements. Thirdly, the properties of each kind of material should be set (properties including: solderability test, tinning, pin shaping), and make the production preparation according to its properties settings. Finally, bake the PCBs and materials for the order.

\subsection{Key technologies}

As shown in Figure 1, the framework of the technology of material management and control of the production preparation execution in SMT production line based on digital recognition conclude the three key technologies below. Its main applications are as follows:

2.2.1. The premise of material management technology is the material management technology based on digital recognition. There are huge number and varieties of material in SMT line, and the production process is relatively complex. Through the rapid identification of the bar code and RFID 
tags, the effective flow of the material in the production process is ensured, and the effective flow control and the related information of the product are realized. This technology can not only make the production preparation process simple and efficient, and it can be used in production processes such as chip mounting, jet printing and AOI inspection to collect PCB information.

2.2.2. The technology of progressive experience base for material property management is the key to assist staff to carry on the material property setting and management. This technology can help the staff to carry out the material property setting problems quickly in production preparation. This technology not only saves a lot of time, but also effectively avoid the lack of property caused by personnel errors.

2.2.3. The technology of execution monitoring base on Kanban system is the core of the implementation of production preparation production execution status monitoring. The SMT-MES monitor Kanban will reflect out production schedule in time. It's convenient for the staff to make the corresponding decision of the production process, and effectively improve the production efficiency of SMT line.

\section{The material management technology based on digital recognition}

In the process of preparation for the SMT production line, in order to make the information flow, material flow, business flow highly unified in MES and achieve orders, material, the current operation, location, time and other information of PCB to be obtained completely, the material management technology based on digital recognition is suggested.

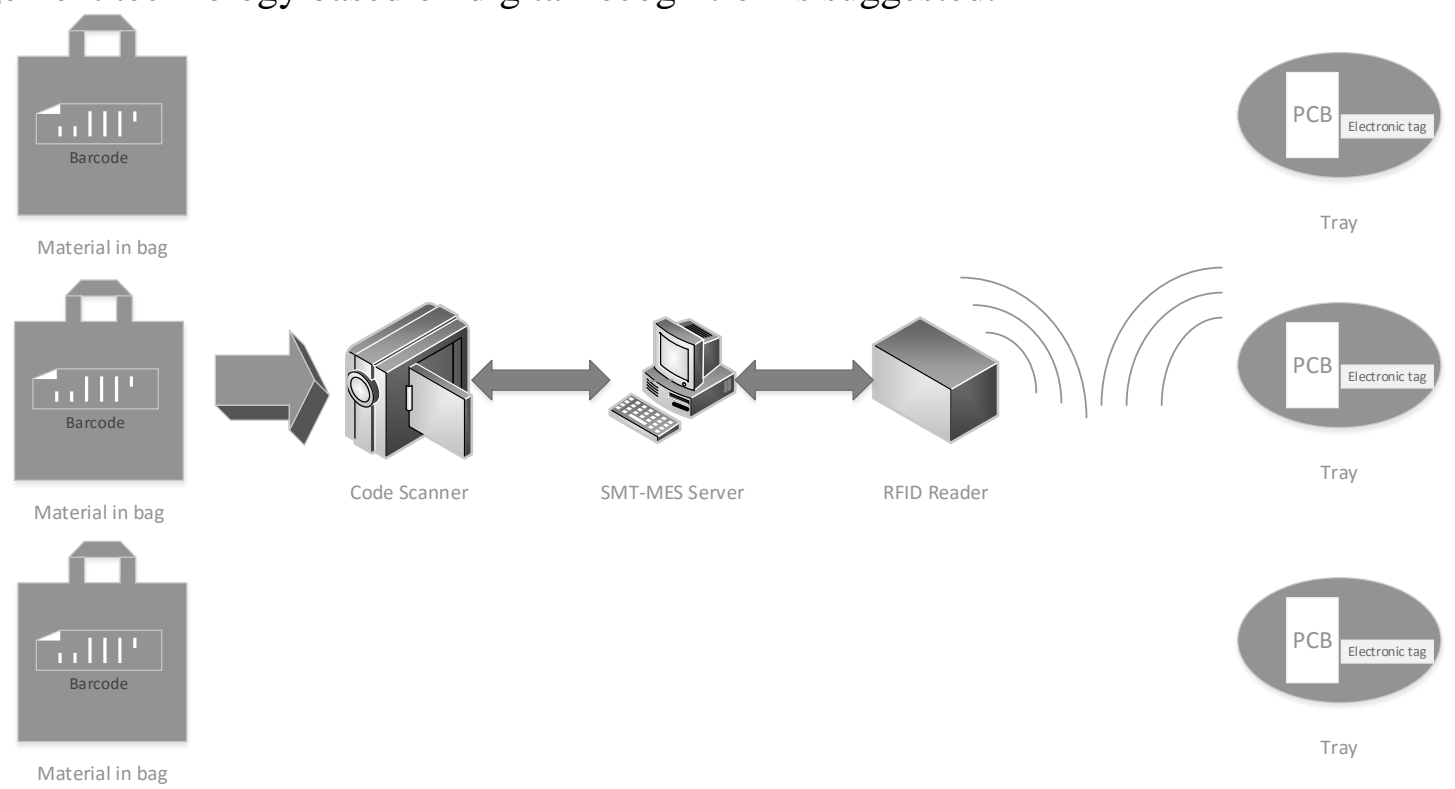

Fig.2 Digital recognition

\subsection{The correlation technology of electronic components information}

In the process of production preparation, the material is the first issue of the follow-up process. The type of material, the number of each material and the number of PCB are the key factors of each order. The problems with these factors will cause downtime, delay and other consequences to the production line. This paper using bar code technology to make the order information, material information, electronic components and PCBs batch information unity in a bar code, realize correlation of the complex material information.

According to the actual situation of SMT production line, the material contains a variety of information, so we choose to use two-dimensional code as the basis of bar code. Because the component is small, it is difficult to distinguish one by one. So before the circulation of materials, we put all materials which all belongs to the same order into a bag, and then in the bag paste bar code identification. In SMT-MES we need to build correlation relationship of orders - material barcode - batch - quantity. 
By means of scanning the barcode we can easily record the log of materials to be put in and out of the storage, and the number of the materials in SMT-MES can easily change by using this technology.

\subsection{The technology of pushing PCB information based on RFID}

As for PCBs, the bar code technology must use barcode gun to scan. It's inconvenient for the high-speed flow of information collection in PCB production process. Therefore, the technology of pushing PCB information based on RFID is suggested to solve this problem.

RFID technology has two major advantages: first, it can achieve a certain degree of remote information reading. As long as the RFID tag into the reader's identifiable range, you can read the information. Second, it can achieve high-speed mobile information reading.

In order not to add extra burden to the process and not to bring quality problems to PCBs, the RFID tag is not directly embedded in the PCB. It will be embedded in the tray. The association between the PCB bar code and the tray electronic tag can be realized and the recycling of the electronic labels can be realized.

\section{The technology of incremental experience base for material property management}

In the SMT production line, materials in production preparation can be divided into Solderability testing components, tinning components and pin shaping components. These three types are fairly three properties of materials, and they are not exclusive independent. It means each kind of material in the order must complete the settings specifically. If you need to manually set the property of each material, there is no doubt that the workload of the staff is greatly increased. Therefore, this paper puts forward the technology of incremental experience base for material property management to solve this problem.

\subsection{The introduction of the experience base}

In the experience base, a kind of material is used as the basic data, and its number is the unique identification of the experience. Each kind of material includes three kinds of properties, and each property has an experience value. The material also has a total experience value. We can judge the material properties by the relationship between the three experience values and the total experience value. Experience base doesn't need to maintain, an automatic experience accumulation will be put into experience base when the staff sets the material property. This technology helps users to set material properties of a new order, so it is also called incremental experience base.

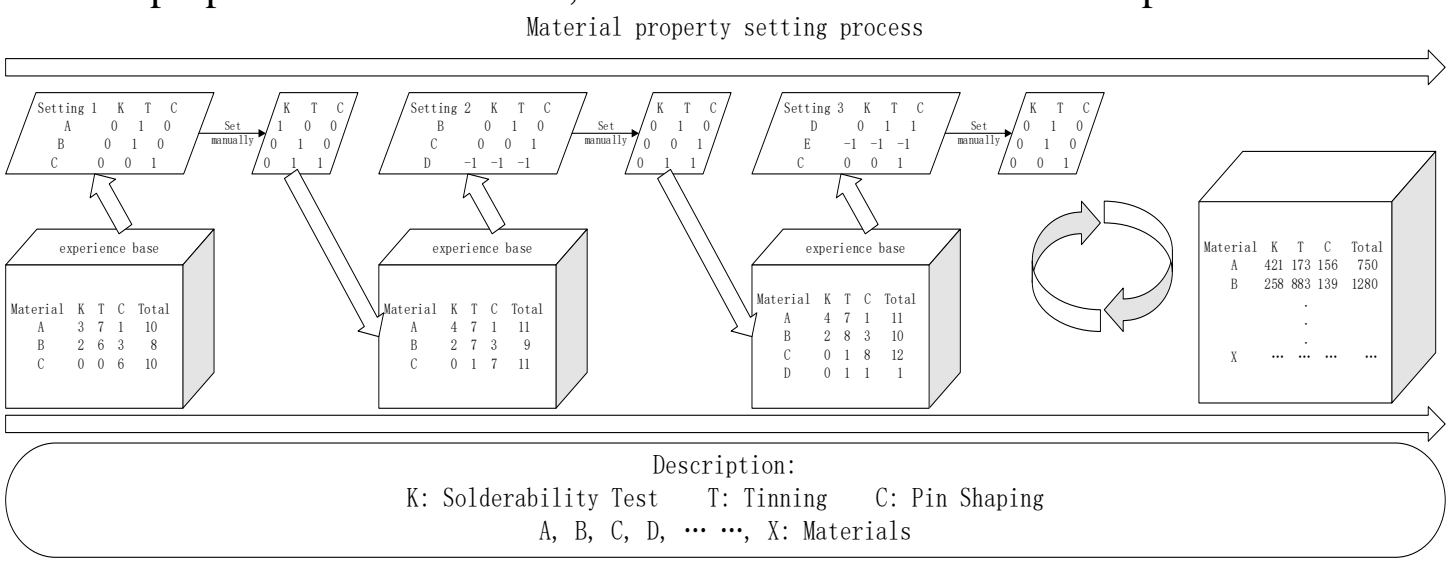

Fig.3 The incremental experience base

\subsection{The rules of the experience base}

The material coding is the sole judgment of the data in the experience base. When the system is used for the first time, there are no data in the experience base. So experience base cannot make judgment of the materials' classification. Therefore, it is necessary for users to set the material properties manually, and the results will be saved into the experience database as empirical data. Every time when user confirm the material property setting, there will be an experience data put into the database. And then according to the material coding to make the sole judgment. If this 
material coding is already exist in experience base, this experience value of the material will get accumulation. If not, the database will create a new experience data. As shown in the second experience base of figure above, after manual modification of the experience of material A from the original $(3,7,1,10)$ add $(1,0,0,1)$ turn into $(4,7,1,11)$. The third experience base add a new experience record of material $\mathrm{D}$.

\subsection{The basis of the experience base judgment}

If the material of the new order task already exists in the experience base, then the experience base will give an empirical judgment to set the properties of the material. Such as the property of material $\mathrm{A}$ in the experience base above including $\mathrm{K}$ (solderability test), $\mathrm{T}$ (tinning) and $\mathrm{C}$ (pin shaping) accumulate experience value is $(3,7,1)$, the total value of the experience data is 10 , make judgment about the K,T,C three properties:

$$
\begin{aligned}
& \mathrm{K} / \text { Total }=3 / 10<0.5, \\
& \mathrm{~T} / \text { Total }=7 / 10>0.5, \\
& \mathrm{C} / \text { Total }=1 / 10<0.5,
\end{aligned}
$$

So the three properties of task 1 are $(0,1,0)$. (The principle is: if $\mathrm{K} /$ Total $>=0.5$ then experience base will determine the value of $\mathrm{K}$ is 1 , otherwise the value will be 0 . And it is the same way for $\mathrm{T}$ and C.)

If the property given by experience base does not meet the requirements of the actual production, the user can manually change it, and the change of the data will be used as a new experience in storage.

\section{The technology of execution monitoring base on Kanban system}

In the preparation process of SMT production line, there are four processes including material kitting, material properties setting, material preparation and baking preparation. For each process, its monitoring is different. Material kitting and baking preparation is take the order as the main line, while the other two processes are mainly based on the materials. This paper suggest the technology of execution monitoring base on Kanban system to realize the refinement of production preparation and implementation of process control.

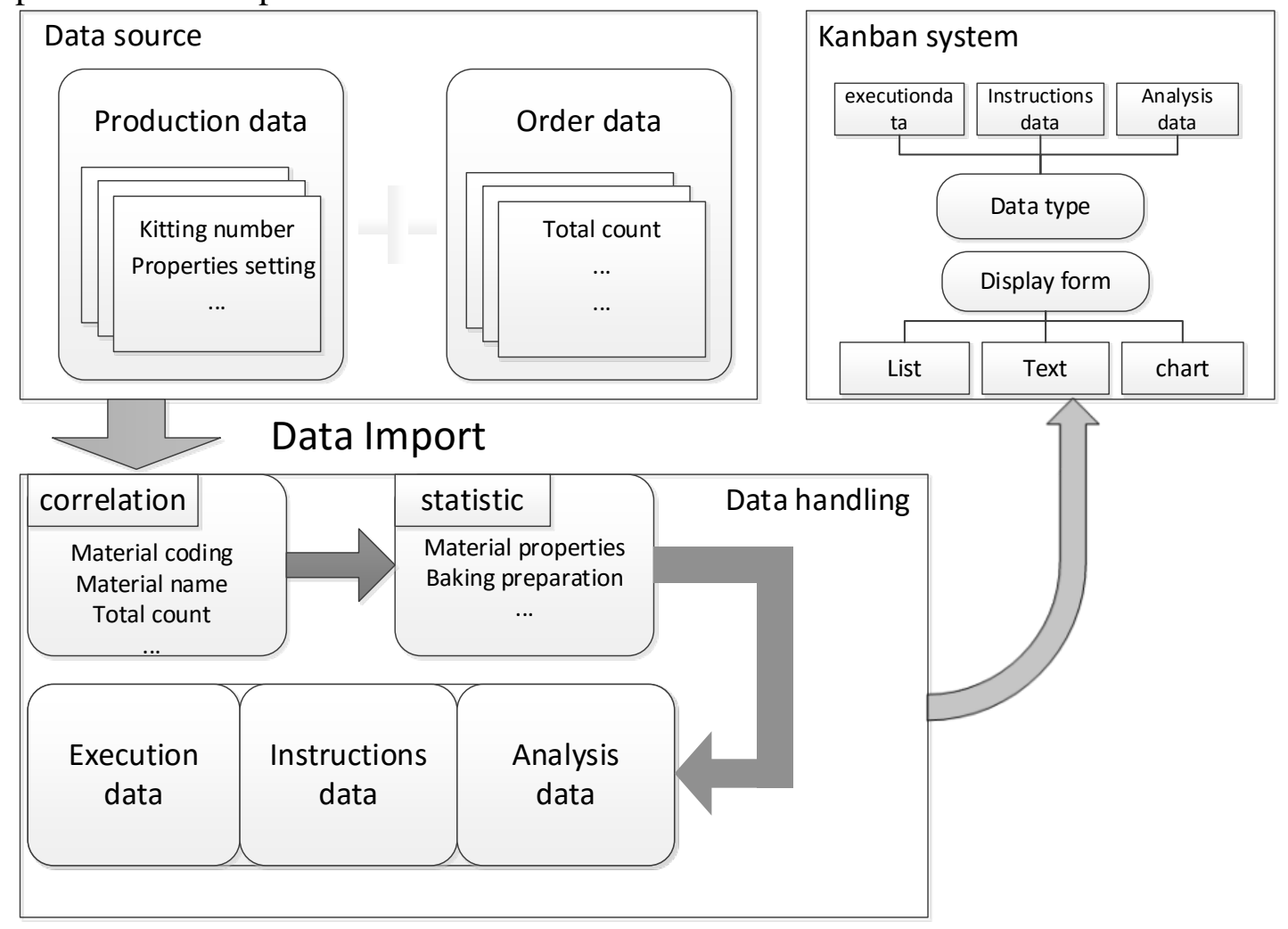

Fig.4 Kanban system

Kanban system is a kind of tool to delivery instructions created by Toyota Motor in the last 
century. The main four functions of Kanban system include instructions of production and operation, prevention of excess production, visual management tools and tool of improving the production process. In the SMT production preparation process, Kanban system mainly plays an important role of reflecting the situation of the workshop and tracking the production information, which enable managers to effectively control the workshop of the actual operation.

All of the business process of the production preparation in SMT can be display in Kanban system. Whether confirming the baking preparation manually or recognizing the material by barcode or recognizing PCB by RFID, these results information could be save into SMT-MES's database, and be used for management decision

\section{Application Verification}

On the basis of applying the technologies above, the author has developed a system for the SMT production process control and the material management. The system successfully achieved the some functions such as information import of material kitting, barcode creating, management of material properties, Kanban system and so on. As shown in figures below.

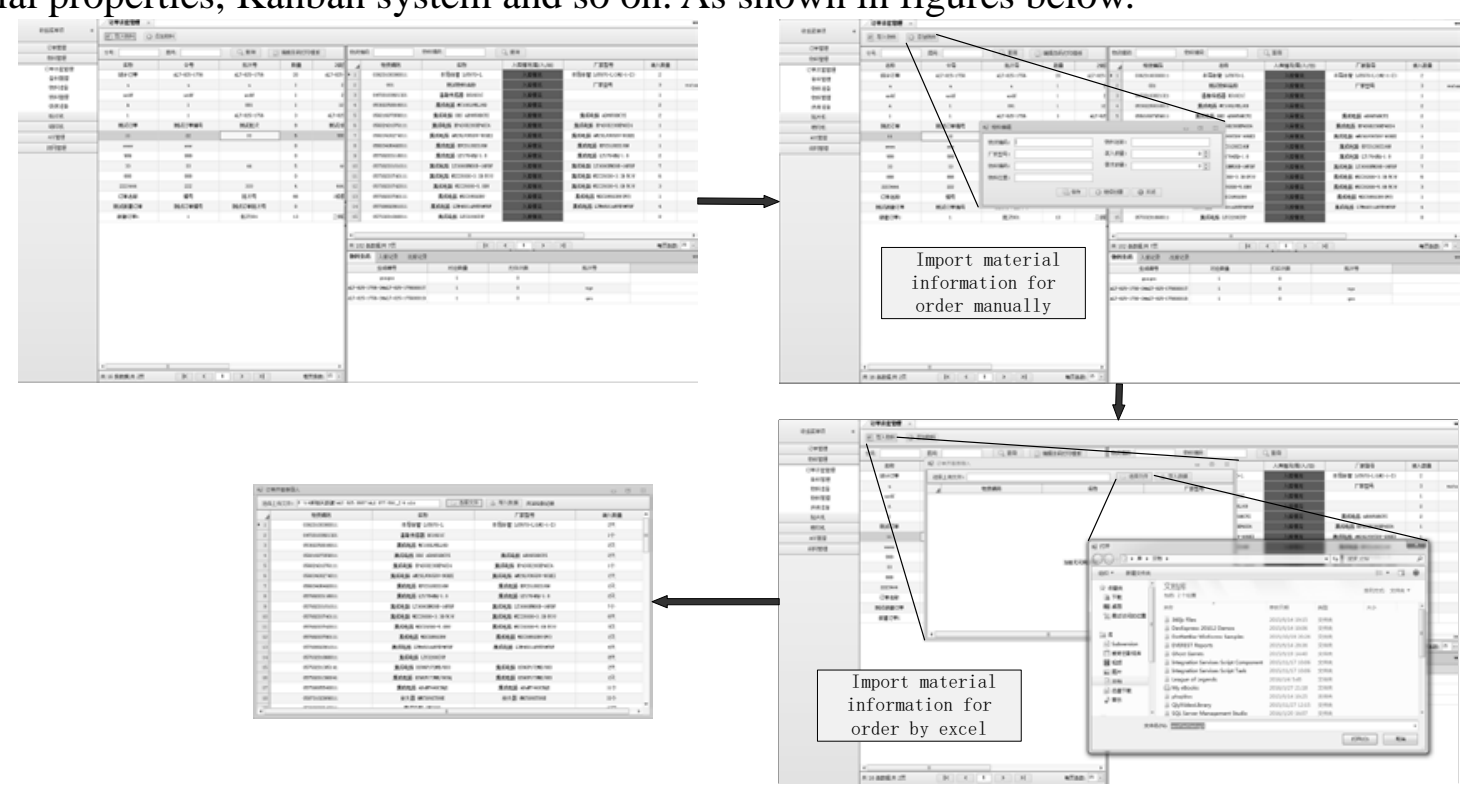

Fig.5 information import of material kitting

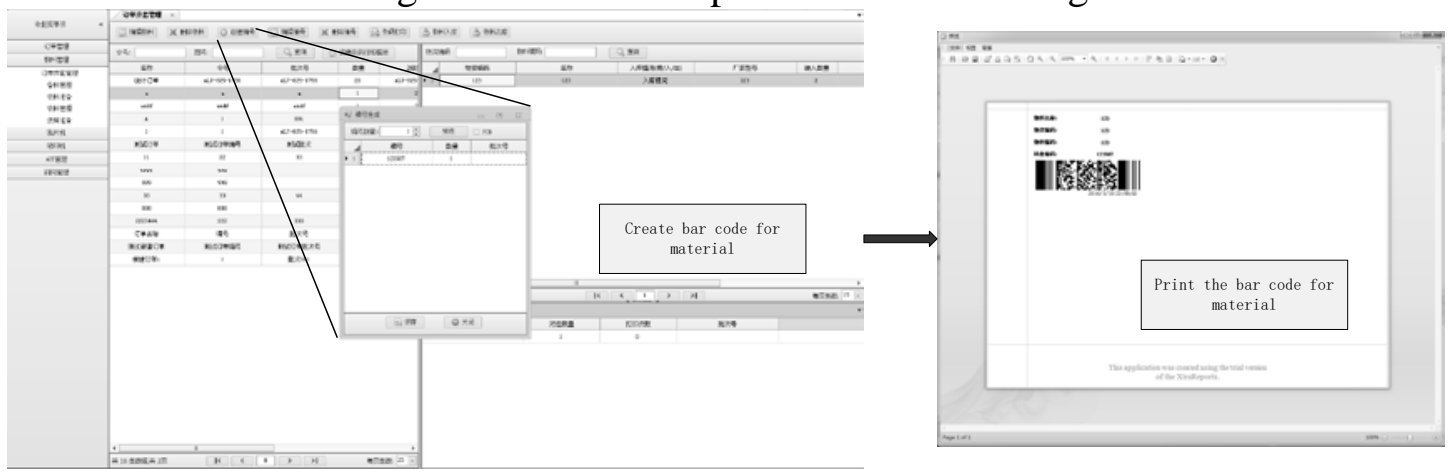

Fig. 6 barcode creating, 

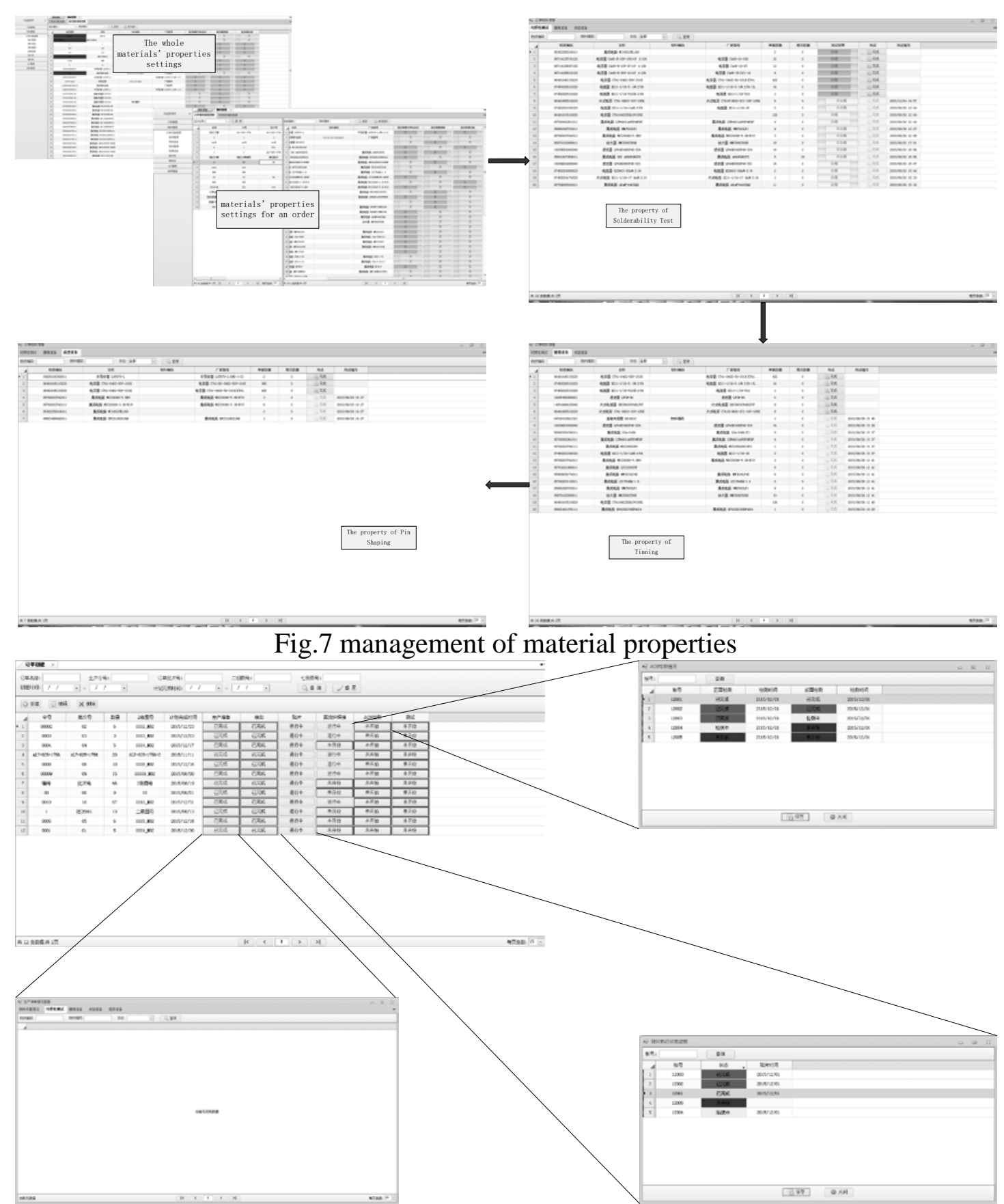

Fig.8 Kanban system

\section{Conclusion}

In this paper, the technology of material management and control of the production preparation execution in SMT production line based on digital recognition is proposed including three key technologies: the material management technology based on digital recognition, the technology of progressive experience base for material property management, the technology of execution monitoring base on Kanban system. And a SMT-MES management system is designed based on this technology. The three key technologies are applied to SMT production line in this system, and it successfully improve the execution control of the SMT production preparation process and management of materials.

\section{References}

[1] Andy Tseng, Mark Lin, Bruce Hu etc. Advanced QFN surface mount application notes development [C]. 2010 12th Electronics Packaging Technology Conference, EPTC 2010,p 
737-742, 2010

[2] Sangchul Kim, Yongwoo Kim. Material management system based on daily work [J]. Advanced Materials Research, v 255-260, pt.3, 1587-91, 2011

[3] WANG Fan, BI Ming-lu, GUO Rui-gang. The design and implementation of management system in SMT workshop [J]. Modern Electronics Technique 2011(7) p182-185

[4] .Zhang Hao.Yu Feng-lian. The research on the manufacturing execution system of SMT workshop based on Intelligent Object [J]. Industrial Engineering Journal 2015(2), p142-150 